\title{
Platelet adhesion changes during storage studied with a novel method using flow cytometry and protein-coated beads
}

Nahreen Tynngård, Maria Wallstedt, Anna L Södergren, Lars Faxälv and Sofia Ramström

\author{
Linköping University Post Print
}

Tweet

N.B.: When citing this work, cite the original article.

Original Publication:

Nahreen Tynngård, Maria Wallstedt, Anna L Södergren, Lars Faxälv and Sofia Ramström, Platelet adhesion changes during storage studied with a novel method using flow cytometry and protein-coated beads, 2014, Platelets.

http://dx.doi.org/10.3109/09537104.2014.891728

Copyright: Informa Healthcare http://informahealthcare.com/

Postprint available at: Linköping University Electronic Press

http://urn.kb.se/resolve?urn=urn:nbn:se:liu:diva-111533 


\section{Platelet adhesion changes during storage studied with a novel method using flow cytometry and protein-coated beads}

NAHREEN TYNNGÅRD ${ }^{1,2}$, MARIA WALLSTEDT ${ }^{2}$, ANNA L SÖDERGREN ${ }^{2}$, LARS FAXÄLV ${ }^{2}, \&$ SOFIA RAMSTRÖM ${ }^{2}$

${ }^{1}$ Transfusion Medicine, Department of Clinical and Experimental Medicine, Faculty of Health Sciences, Linköping University; Department of Clinical Immunology and Transfusion Medicine, County Council of Östergötland, Linköping, Sweden

${ }^{2}$ Clinical Chemistry, Department of Clinical and Experimental Medicine, Faculty of Health Sciences, Linköping University; Department of Clinical Chemistry, County Council of Östergötland, Linköping, Sweden

Correspondence to: Sofia Ramström
Department of Clinical and Experimental Medicine,
Clinical Chemistry, Faculty of Health Sciences
Linköping University
SE-581 85 Linköping, Sweden
Telephone +46101038600 Fax +461010333240
e-mail: sofia.ramstrom@liu.se

Short title: Platelet adhesion to protein-coated beads

Key words: Adhesion molecules, blood products, transfusion medicine, platelet glycoproteins 


\begin{abstract}
The aim of the present study was to set up and evaluate a novel method for studies of platelet adhesion and activation in blood and platelet suspensions such as platelet concentrate (PC) samples using protein-coated polystyrene beads and flow cytometry. To demonstrate its usefulness, we studied PCs during storage. PCs were prepared by apheresis technique $(N=7)$. Metabolic variables and platelet function was measured on day 1, 5, 7 and 12 of storage. Spontaneous and TRAP-6-induced adhesion to fibrinogen- and collagen-coated beads was analysed by flow cytometry. P-selectin and phosphatidyl serine (PS) expression was assessed on platelets bound to beads as well as on non-adherent platelets. Platelet adhesion to fibrinogen beads had increased by day 12 and adhesion to collagen beads at day 7 of storage $(p<0.05)$. TRAP-6 stimulation significantly increased the platelet adhesion to fibrinogen beads $(p<0.05)$ as well as the P-selectin and PS exposure on platelets bound to beads $(p<$ 0.01) during the first 7 days of storage, but by day 12, significant changes were no longer induced by TRAP-6 stimulation.

We demonstrate that our adhesion assay using protein-coated polystyrene beads can be used to assess the adhesion properties of platelets during storage without the addition of red blood cells. Therefore it may offer a useful tool for future studies of platelet adhesive capacity in transfusion medicine and other settings.
\end{abstract}




\section{Introduction}

Platelets have an important role in the haemostatic process. In order to seal damaged blood vessels, platelets will adhere to proteins exposed in the subendothelium. The adhesion process involves several platelet surface receptors. At high shear conditions, platelets will bind to von Willebrand factor (vWF) immobilised on exposed collagen in the vessel wall through glycoprotein (GP)Ib/IX/V receptor complex, followed by platelet activation when GPVI binds to collagen. Firm adhesion and spreading is mediated by GPIIb/IIIa binding to vWF, fibrinogen and fibronectin and GPIa/IIa binding to collagen [1,2]. This results in platelet activation, shape change, secretion from granule and exposure of phosphatidylserine (PS).

It is important that platelets used for transfusion have good haemostatic ability. However, storage of platelet concentrates (PCs) results in the platelet storage lesion (PSL) including changes in metabolism, surface receptors and response towards agonists, as reviewed by Cauwenberghs et al. and Ohto et al. [2,3]. Quality testing of PCs often involves analysis by several in vitro methods, including analysis of blood gases, metabolic variables, activation markers, and the more novel technique of analysing aggregation using impedance aggregometry (i.e. Multiplate) $[4,5]$. However, there are few studies that investigate the function of platelets in terms of adhesion capacity. One reason is that current available methods are time consuming, complicated to use, and require large sample volumes. The adhesion capacity of stored platelets has been studied using perfusion chambers [6-8] or cone and-plate devices $[9,10]$. These methods require the presence of red blood cells, which complicates the analysis of PCs and introduces a potential source of non-specific and unpredictable effects $[8,9,11]$. As an example, it has been shown that the age of red blood cells used for suspension of platelets from PCs could affect the adhesion [12]. The relevance 
of the presence of red blood cells when assessing platelet function in vitro on the effects of on the in vivo function is unclear.

The aim of the present study was to set up and evaluate an easy and fast method using protein-coated polystyrene beads and flow cytometry to facilitate investigation and quantification of platelet adhesion in both whole blood and platelet suspensions without the use of pumps or microscopes. This new method was evaluated by studying the adhesion capacity of platelets in PCs to fibrinogen and collagen beads during prolonged storage in parallel with other in vitro methods. 


\section{Methods}

The study was approved by the Ethics Committee at Linköping University Hospital.

\section{Bead coating}

$20 \mu \mathrm{L}$ of polystyrene bead solution $(20 \mu \mathrm{m}$ in diameter, $10 \%$ wt solution; Sigma-Aldrich Co., St. Louis, MO, USA), was incubated with $80 \mu \mathrm{L}$ of different protein solutions for 30 minutes at room temperature; bovine serum albumin (BSA, final concentration $0.8 \mathrm{mg} / \mathrm{mL}$; SigmaAldrich Co.), fibrinogen (final concentration $8 \mathrm{mg} / \mathrm{mL}$; Sigma-Aldrich Co.) or collagen Horm (final concentration $80 \mu \mathrm{g} / \mathrm{mL}$; Multiplate COLtest, Verum Diagnostica GmbH, Munich, Germany). The beads were aliquoted and stored at $4{ }^{\circ} \mathrm{C}$ (collagen beads) or at $-80^{\circ} \mathrm{C}$ (BSA and fibrinogen beads).

\section{Blood collection}

Venous blood was collected by venipuncture from healthy adult volunteers. Blood was collected and anticoagulated with heparin (final concentration 11-17 U/mL).

\section{Platelet collection, preparation, storage and sampling}

Platelets were collected by standard apheresis procedures (COBE Spectra v.7.0 LRS Turbo and Trima Accel v.5.0 LRS, Terumo BCT, Lakewood, CO, USA) from 7 healthy donors. ACD (Terumo BCT) was used as anticoagulant agent [13]. The platelet concentrates (PCs) contained in average $298 \pm 37 \times 10^{9}$ platelets on the day of donation (day 0). All PCs were irradiated with 25 Gy on day 0 (Gammacell 3000 Elan, MDS Nordion, Ottawa, Canada) according to routine procedures at the Department of Clinical Immunology and Transfusion Medicine in Linköping. The PCs were stored in bags integrated to each collection kit for the cell separators on an agitator (Model LPR-3, Melco Engineering, Glendale, CA, USA) in an 
incubator (Helmer PC2200, Helmer, Noblesville, IN) with a stable temperature of $22 \pm 2{ }^{\circ} \mathrm{C}$. A sample was taken aseptically on day 1, 5, 7 and 12 from each PC with a sampling bag (Maco Pharma, Tourcoing, France) and used for various in vitro tests (see below). For flow cytometry, the platelet concentration was adjusted to $300 \times 10^{9}$ platelets/L by dilution with HEPES buffer. The dilution of the sample for flow cytometry on day 1, 5, 7 and 12 was based on the platelet concentration determined on day 0 .

\section{Examination of basal PC parameters}

Swirling of the PCs was examined by visual inspection [14] and graded as $0,+,++$ or $+++(0$ $=$ no swirling,$+++=$ maximum swirling) according to routine procedures at the Department of Clinical Immunology and Transfusion Medicine in Linköping. Platelet concentration and mean PLT volume (MPV) were measured with the Cell-Sapphire hematology analyser (Abbott Diagnostics Division, Abbot Park, IL, USA). $\mathrm{pH}$ was measured at $37^{\circ} \mathrm{C}$ with a blood gas analyser (Cobas b221, Roche, Diagnostics GmbH, Mannheim, Germany). Glucose, lactate and lactate dehydrogenase (LDH) were measured in autologous plasma with a chemistry analyzer (Advia 1800, Siemens AG, Erlangen, Germany).

\section{Expression of GPIb, P-selectin and phosphatidylserine (PS) on single platelets in PCs}

The expression of the surface receptor GPIb, activation marker P-selectin and PS, a potential marker for apoptosis, was analysed by flow cytometry on a Gallios flow cytometer (Beckman Coulter Inc., Fullerton, CA, USA). Fluorosphere control samples (Flow-Check and Flow-Set, Beckman Coulter Inc.) were used to verify that the instrument performance was stable over time. Expression of GPIb was analysed as previously described [13]. P-selectin and PS exposure was analyzed by adding $3 \mu \mathrm{L}$ of platelet suspension, diluted as described above, to $33 \mu \mathrm{L}$ of antibody solution in HEPES buffer (137 mM NaCl, $2.7 \mathrm{mM} \mathrm{KCl}, 1 \mathrm{mM} \mathrm{MgCl} 2,5.6$ 
$\mathrm{mM}$ glucose, $1 \mathrm{~g} / \mathrm{L}$ bovine serum albumin and $20 \mathrm{mM}$ HEPES, $\mathrm{pH}$ 7.40) supplemented with $2.5 \mathrm{mM}$ calcium. The antibody solution consisted of anti-CD41a-PE (final concentration 0.03 $\mu \mathrm{g} / \mathrm{mL}$; BD Biosciences, San Jose, CA, USA) and anti-CD62P-PECy5 (P-selectin, final concentration $1 \mu \mathrm{g} / \mathrm{mL}$; BD Biosciences) or annexin V-FITC (final concentration $0.1 \mu \mathrm{g} / \mathrm{mL}$; BD Biosciences) for PS expression. As negative control for P-selectin, PECy5 isotype antibody (final concentration $1 \mu \mathrm{g} / \mathrm{mL}$; BD Biosciences) was used. As a negative control for annexin V binding, HEPES buffer without calcium was used in all steps throughout the procedure for those samples, as the lack of calcium will prevent annexin $\mathrm{V}$ binding to platelets $[15,16]$. Samples were incubated for $10 \mathrm{~min}$ in the dark, followed by dilution 1:20 with the same buffer as for the incubation step. Platelet detection was based on anti-CD41aPE fluorescence and forward scatter properties [17] and P-selectin or PS exposure presented as percentage of PECy5+ or FITC+ platelets, respectively, with the marker in the negative control samples set to $1-2 \%$ positive platelets [18].

\section{Platelet adhesion to beads of platelets in whole blood and PCs}

The adhesion of platelets to beads coated with fibrinogen or collagen was assessed by flow cytometry, beads coated with BSA were used as control. Spontaneous platelet adhesion to beads was assessed as well as adhesion of platelets stimulated with ADP or a peptide activating the platelet thrombin receptor PAR-1 (TRAP-6, amino acid sequence SFLLRN) when platelets in whole blood was analysed. Spontaneous platelet adhesion as well as TRAP6 induced adhesion was assessed on platelets from PCs.

For the platelet inhibition tests, whole blood samples were pre-incubated with buffer, the $\mathrm{P} \mathrm{Y}_{12}$ ADP receptor inhibitor cangrelor (final concentration $100 \mu \mathrm{M}$; The Medicines Company, Parsippany, NJ) or the fibrinogen receptor inhibitor abciximab (Reopro, final 
concentration $20 \mu \mathrm{g} / \mathrm{mL}$; Eli Lilly \& Co., Indianapolis, IN) for $20 \mathrm{~min}$ before adding the blood to the bead suspension tubes. The expression of P-selectin and PS was assessed on platelets from PC samples that had adhered to beads.

$10 \mu \mathrm{L}$ of well mixed bead solution was added to $65 \mu \mathrm{L}$ of filtered HEPES buffer, supplemented with FITC-conjugated chicken antibodies against P-selectin (final concentration $55 \mu \mathrm{g} / \mathrm{mL}$; Diapensia HB, Linköping, Sweden) and when required, TRAP-6 (final concentration $30 \mu \mathrm{M}$; JPT, Berlin, Germany) or ADP (final concentration $10 \mu \mathrm{M}$; Sigma-Aldrich Co.). $1 \mu \mathrm{L}$ of well mixed whole blood or PC (diluted as described above) was added and the tubes were vortexed at $2000 \mathrm{rpm}$ for $30 \mathrm{~s} .1 \mu \mathrm{L}$ anti-GPIIb-PE (clone 5B12), final concentration $1 \mu \mathrm{g} / \mathrm{mL}$, recognizing both activated and resting receptor, and not affected by abciximab; Dako AS, Glostrup, Denmark) was added and the tubes were incubated for 5 min in the dark followed by addition of $500 \mu \mathrm{L}$ of HEPES buffer and a gentle vortex to stop the reactions. For the PS samples, annexin V-FITC (final concentration $0.1 \mu \mathrm{g} / \mathrm{mL}$ ) and a calcium-supplemented buffer (140 mM NaCl, $2.5 \mathrm{mM} \mathrm{CaCl}_{2}$ and $10 \mathrm{mM}$ HEPES, pH 7.40) was used.

The whole blood samples were analysed on a Coulter Epics XL MCL flow cytometer (Beckman Coulter Inc.). The PC samples were analysed on the recently introduced Gallios flow cytometer (Beckman Coulter Inc). Platelets and beads were identified based on their specific forward and side scatter properties. Data was collected for $180 \mathrm{~s}$ at high speed to collect enough beads to allow reliable analysis. Samples showing $<1000$ beads or $<5000$ single platelets were considered erroneous and excluded from further analysis. Single beads were gated and assessed for platelet adhesion. Anti-GPIIb-PE was used to detect platelets bound to beads. Platelet adhesion to beads was determined as the percentage of GPIIb- 
positive single beads. Beads with platelets were also assessed for the amount of platelet adhesion to each bead (X-median PE). The gating strategy is summarised in Figure 1. Beads with adhering platelets (i.e. stained positive for GPIIb as shown in the histogram plot in Figure 1) were gated and the expression of PS and P-selectin quantified. Annexin V binding is presented as the percentage of annexin $\mathrm{V}+$ platelets as the $\mathrm{X}$-median FITC fluorescence for annexin $\mathrm{V}$ was too low to allow reliable evaluation. Expression of P-selectin is presented as $\mathrm{X}$-median FITC fluorescence. 



Figure 1

Figure 1. Gating and analysis strategy for measuring platelet adhesion and activation to protein-coated polystyrene beads by flow cytometry.

The dot plot on the left shows detected platelets, white and red blood cells and beads. The Beads gate is further evaluated to exclude multiple bead events before further analysis. The histogram on the right shows populations of single beads with and without adhered platelets, as detected by the presence of GPIIb-PE antibody. Beads with adhered platelets are gated as shown in the graph and further evaluated for FITC fluorescence (expression of P-selectin/PS) and median PE fluorescence (number of platelets attached to each bead) in a histogram.

\section{Statistics}

The results are presented as mean \pm standard deviation (SD). Potential changes between days and treatments were evaluated by repeated measures ANOVA with Bonferroni's multiple comparisons test using GraphPad Prism (version 5.02, GraphPad software Inc., San Diego, 
CA, USA). A $p$-value of $<0.05$ was considered significant. Due to technical difficulties with some PC samples and one PC with only minimal swirling on day 12, the number of PCs included in the statistical evaluation is 5 for BSA and collagen beads and 6 for fibrinogen beads, as the statistical method does not allow series with missing values. However, all available data points are represented in the figures, except for the day 12 results for the unit with minimal swirling. The data on day 12 for the PC with minimal swirling is presented separately not to affect the statistics as this PC was exceptional in this characteristic. 


\section{Results}

\section{Validation of the bead adhesion assay}

Stability and variability

No major differences in platelet adhesion were observed between different batches of proteincoated beads, for beads stored for up to 4 months, or between fresh and frozen fibrinogen beads. Freshly prepared fibrinogen beads showed $25 \%$ platelet adhesion as did 3months old beads. One month old collagen beads showed $25 \%$ platelet adhesion and 4 months old $28 \%$. To avoid pre-analytical loss of beads, they were not washed after the coating procedure. However, we confirmed that the coating solution itself did not induce platelet activation or aggregation (data not shown). Assessing the P-selectin expression on non-adhered platelets in the bead samples confirmed that the beads did not induce platelet activation. We found that only $4-5 \%$ of the non-adhered platelets from one day old PCs expressed P-selectin in presence of the different bead types. The coefficient of variation (CV) for platelet adhesion in duplicate samples of whole blood was $20 \%$ for BSA and fibrinogen beads and $8 \%$ for collagen beads ( $N$ $=15-30)$. The CV for platelet adhesion for duplicate samples of PCs was $10 \%$ for fibrinogen beads $(N=28)$ and $15 \%$ for collagen beads $(N=25)$. To evaluate the precision in pipetting, we also calculated the variability in bead number and number of single platelets in duplicate BSA bead samples, where low platelet adhesion would be expected. Here, we found the CV to be $7 \%$ for bead number and $4 \%$ for number of single platelets $(N=6)$.

\section{Platelet adhesion in whole blood}

As shown in Figure 2A, platelet adhesion to beads coated with BSA was consistently low, with $0.9 \pm 0.5 \%$ of the beads positive for GPIIb $(N=5)$. Beads coated with collagen (Fig. 2B) showed a moderate platelet adhesion $(17.7 \pm 9.2 \%, N=5)$. For BSA and collagen beads, no 
significant changes were observed when platelets were activated by TRAP- 6 or inhibited by abciximab or cangrelor.
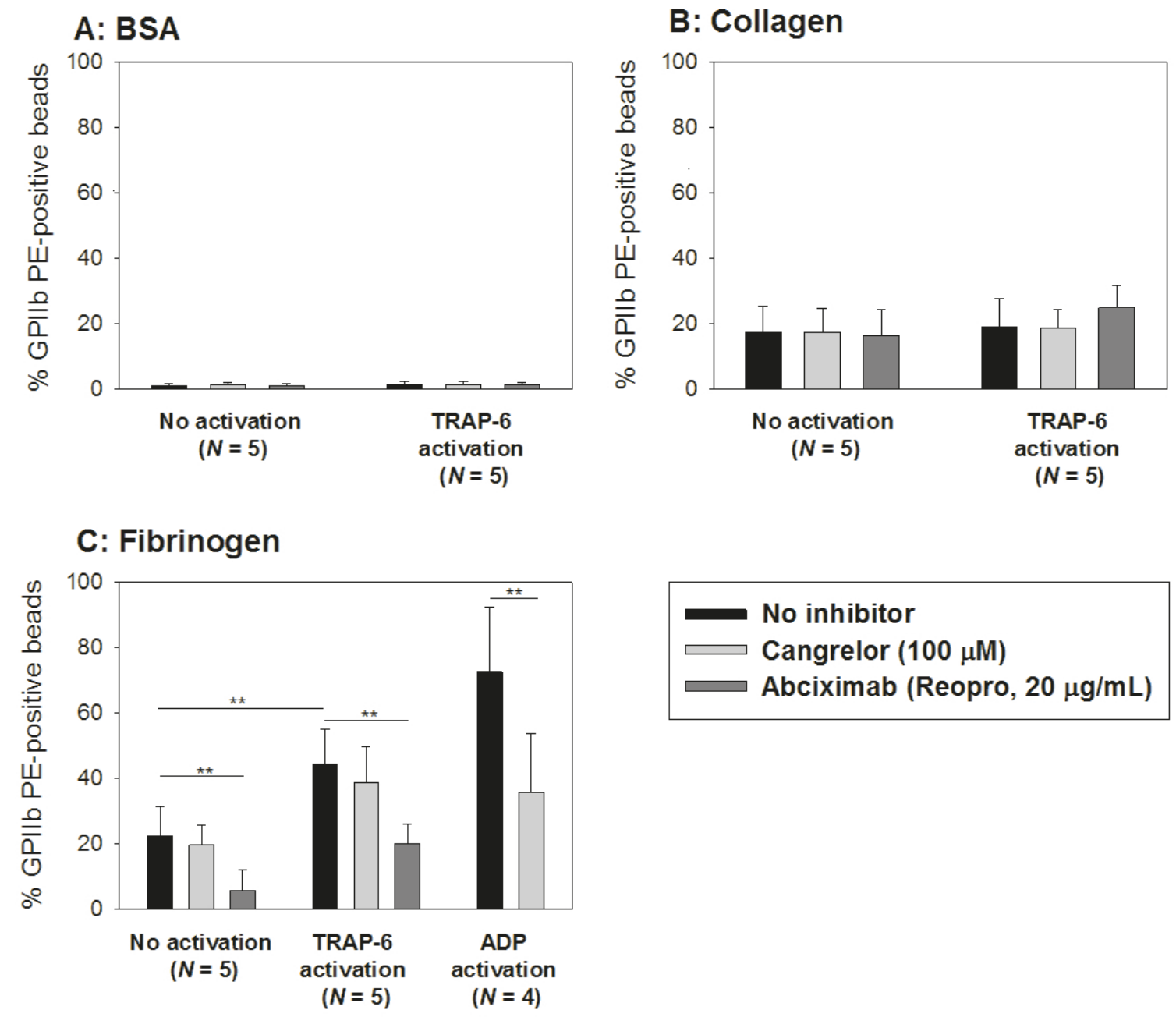

Figure 2. Effect of platelet activation and inhibition on platelet adhesion to beads in whole blood.

The beads were coated with (A) BSA, (B) collagen and (C) fibrinogen. Beads with adherent platelets were defined as beads being positive for the platelet marker GPIIb. The samples were either non-activated or activated by TRAP-6 (SFLLRN, $30 \square \mathrm{M}$ ). For fibrinogen beads, activation with ADP (10 $\square \mathrm{M})$ was also tested. The bars show mean $+\mathrm{SD}(N=5$, except ADP activation, where $N=4$, ** indicates $p<0.01)$.

Platelet adhesion to fibrinogen beads was $24.7 \pm 9.0 \%$, but in contrast to the other bead coatings, adhesion increased significantly following platelet activation by TRAP-6 $(p<0.01)$ and decreased when platelets were inhibited by abciximab $(p<0.01, N=5)$. Adhesion to the 
fibrinogen beads in the presence of ADP was significantly decreased in the presence of cangrelor, as shown in Figure $2 \mathrm{C}(p<0.01, N=4)$. The flow cytometry findings were also confirmed by parallel examination of samples using phase contrast and fluorescence microscopy, beads incubated with BSA were rarely found with attached platelets, whereas beads with fibrinogen and collagen were frequently found with adherent platelets (Figure 3). 


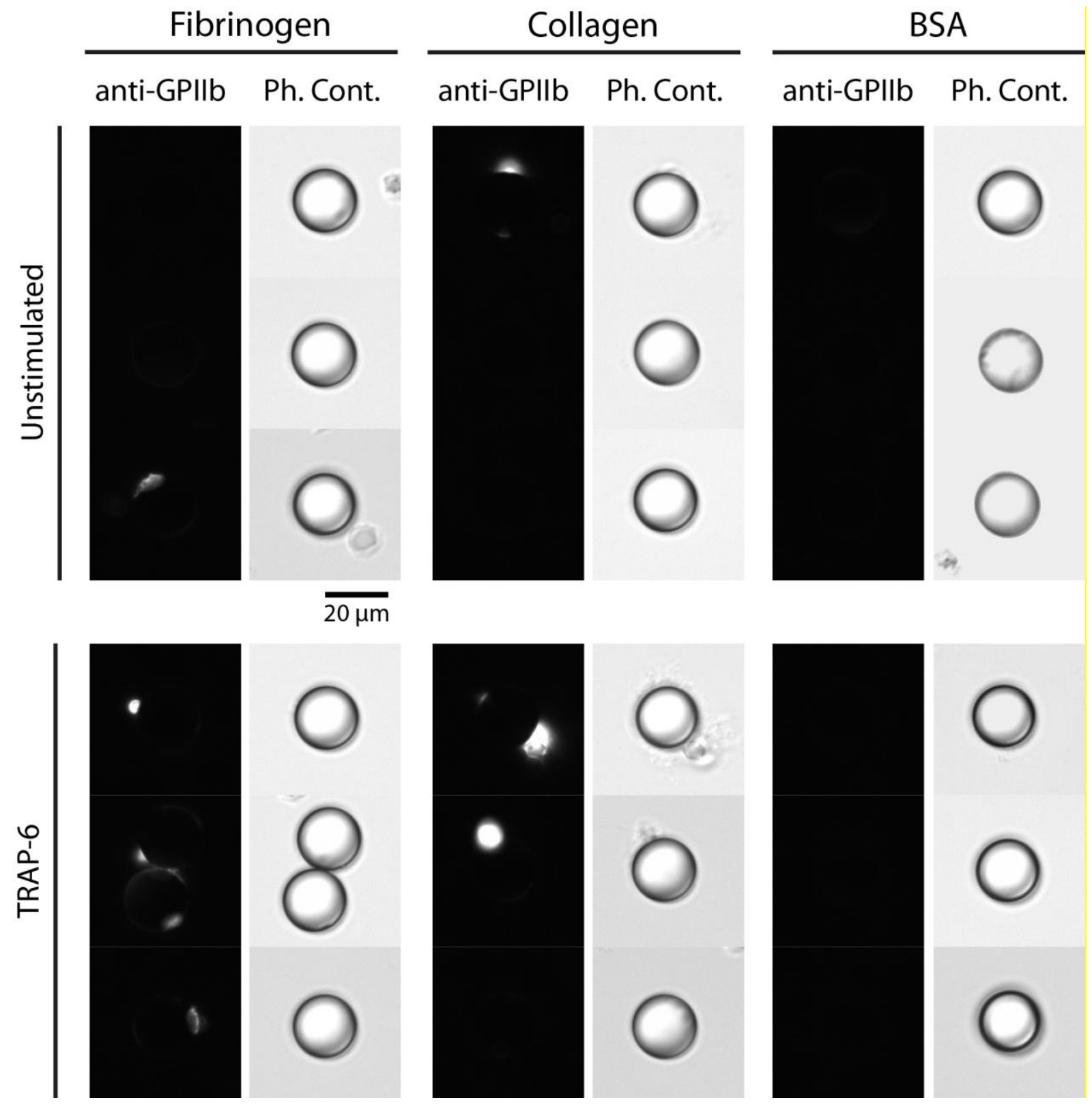

Figure 3. Evaluation of adhesion of platelets from whole blood to protein-coated beads by microscopy. Fluorescence (anti-GPIIb) and phase contrast ( $\mathrm{Ph}$ Cont.) images of beads prepared for flow cytometry were captured using a fluorescence microscope. Approximately 20 beads in each category were investigated for platelet binding. Three beads from each coating preparation, exposed to unstimulated or TRAP-6-activated platelets are shown as a qualitative verification of platelet binding. None of the displayed BSA beads had bound platelets. The $1 / 3$ of the displayed fibrinogen beads of unstimulated samples bound platelets and $1 / 3$ of the collagen beads. $3 / 3$ of the displayed fibrinogen beads of TRAP- 6 stimulated samples bound platelets and $2 / 3$ of the collagen beads. 


\section{PC storage study results}

PC characteristics during storage

All PCs had well maintained (+++) swirling until Day 7, but on day 12, one PC showed minimum swirling (+). The result of this PC on day 12 is therefore presented separately. The changes in PC characteristics during storage are presented in Table I. pH remained at an acceptable level (> 6.4) throughout storage. The glucose consumption rates between day 1-7 and day 7-12 were $0.061 \pm 0.013$ and $0.074 \pm 0.003 \mathrm{mmol} / 10^{11}$ platelets/day, respectively $(p<$ 0.05). The lactate formation rates between day 1-7 and day $7-12$ were $0.117 \pm 0.039$ and $0.141 \pm 0.022 \mathrm{mmol} / 10^{11}$ platelets/day, respectively $(p<0.05)$. The unit with minimal swirling on day 12 had a pH below the acceptable level. This unit had lowest glucose level in the beginning of the study and it was depleted by day 12 (Table I).. 
Table I. PC characteristics

\begin{tabular}{|c|c|c|c|c|}
\hline Parameter & Day 1 & Day 5 & Day 7 & Day 12 \\
\hline $\begin{array}{l}\text { PLT concentration } \\
\left(\mathrm{x} 10^{9} / \mathrm{L}\right)\end{array}$ & $1305 \pm 120$ & $1396 \pm 126$ & $1341 \pm 116$ & $1298 \pm 124(1392)$ \\
\hline $\begin{array}{l}\text { PLT number } \\
\text { ( } \times 10^{9} / \text { unit) }\end{array}$ & $294 \pm 32$ & $295 \pm 38$ & $264 \pm 45^{* *}$ & $245 \pm 52 * *(192)$ \\
\hline $\begin{array}{l}\text { Volume } \\
(\mathrm{mL})\end{array}$ & $227 \pm 34$ & $213 \pm 39 * *$ & $199 \pm 41^{* *}$ & $189 \pm 39 * *$ ๆ $(138)$ \\
\hline $\begin{array}{l}\text { MPV } \\
\text { (fL) }\end{array}$ & $6.5 \pm 0.8$ & $6.6 \pm 0.8$ & $7.0 \pm 1.3$ & $7.2 \pm 0.4(8.9)$ \\
\hline $\mathrm{pH}$ & $7.35 \pm 0.03$ & $7.34 \pm 0.06$ & $7.17 \pm 0.30$ & $6.96 \pm 0.15^{* *}(6.02)$ \\
\hline $\begin{array}{l}\text { Lactate } \\
(\mathrm{mmol} / \mathrm{L})\end{array}$ & $3.5 \pm 0.8$ & $10.0 \pm 2.4^{* *}$ & $12.8 \pm 2.9 * *$ & $23.0 \pm 3.8 *$ * \\
\hline $\begin{array}{l}\text { Glucose } \\
(\mathrm{mmol} / \mathrm{L})\end{array}$ & $16.5 \pm 0.9$ & $13.3 \pm 1.5^{* *}$ & $11.7 \pm 2.0^{* *}$ & $6.8 \pm 1.8^{* * \boldsymbol{\top}}(0.0)$ \\
\hline $\begin{array}{l}\mathrm{LDH} \\
(\mu \mathrm{kat} / \mathrm{L})\end{array}$ & $2.7 \pm 0.8$ & $3.1 \pm 1.3$ & $2.4 \pm 0.3$ & $2.8 \pm 0.4(4.9)$ \\
\hline
\end{tabular}

Results are presented as mean $\pm \mathrm{SD}$ for $N=7$ except on Day 12 where $N=6$. The result for the PC with minimal swirling on day 12 is shown within parentheses. $* *$ indicates $p<0.01$ as compared to day 1 . $\mathbf{\Upsilon}$ indicates $p<0.01$ for day 7 vs. day 12 .

Expression of GPIb, P-selectin and PS on single platelets from PCs during storage

The expression of GPIb remained high throughout storage (Table II). P-selectin expression increased significantly with time (Table II). The PS exposure also increased during storage but it was only significantly higher on day 12 (Table II). The unit with minimal swirling on day 12 had lower expression of GPIb, higher expression of P-selectin and PS than the other units (Table II). 
Table II. Expression of GPIb, P-selectin and PS on single platelets in PCs

\begin{tabular}{|c|c|c|c|c|}
\hline Parameter & Day 1 & Day 5 & Day 7 & Day 12 \\
\hline $\begin{array}{l}\text { GPIb } \\
(\%)\end{array}$ & $91 \pm 8$ & $97 \pm 1$ & $97 \pm 1$ & $93 \pm 3(88)$ \\
\hline $\begin{array}{l}\text { GPIb } \\
\text { (X-median; AU) }\end{array}$ & $34 \pm 5$ & $34 \pm 6$ & $40 \pm 10^{* *}$ & $31 \pm 9$ ฯ (16) \\
\hline $\begin{array}{l}\text { P-selectin } \\
(\%)\end{array}$ & $26 \pm 9$ & $55 \pm 9 * *$ & $82 \pm 12^{* *}$ & $80 \pm 11^{* *}(96)$ \\
\hline PS & $2 \pm 1$ & $3 \pm 1$ & $4 \pm 1$ & $9 \pm 2 * * \boldsymbol{\top}(43)$ \\
\hline
\end{tabular}

Results are presented as mean $\pm \mathrm{SD}$ for $N=6$ for P-selectin and PS and $N=7$ for GPIb except on Day 12 where $N=5$ for P-selectin and PS and $N=6$ for GPIb. The result for the PC with minimal swirling on day 12 is shown within parentheses. ${ }^{* *}$ indicates $p<0.01$ as compared to day $1 . \boldsymbol{\Phi}$ indicates $p<0.05$, $\boldsymbol{\Upsilon}$ indicates $p<0.01$ for day 7 vs. day 12 .

Platelet adhesion to beads in PCs during storage

The platelet adhesion to fibrinogen and collagen-coated beads is shown in Figure 4. Adhesion was assessed as percentage of beads with bound platelets (\% GPIIb-PE-positive beads) and the amount of platelets bound to the beads (X-median PE on beads with platelets) with and without activation by TRAP-6. Spontaneous platelet adhesion to BSA beads was consistently low throughout the study $(2.6 \pm 1.5 \%)$. The percentage of beads with adhering platelets increased with storage for fibrinogen beads $(p<0.05$ at day 12, Figure 4) but was unchanged for collagen beads (Figure 4). However, the median fluorescence of platelets on collagen beads increased with storage to day $7(p<0.05)$. TRAP-6 stimulation increased the adhesion of platelets to fibrinogen beads, with significant increases in percentage of beads with 
platelets and in the amount of platelets adhering to the beads until day 7 ( $p<0.05$ as compared to non-activated platelets, Figure 4). For the collagen beads, TRAP-6 did not cause any increase in the percentage of beads with adhering platelets, while the median fluorescence increased slightly, although not significantly (Figure 4B). On day 7, the percentage of fibrinogen beads with adhering platelets following TRAP-6 stimulation was significantly higher than on day $1(p<0.05)$, but on day 12 the response had decreased to a level similar as on day 1. The TRAP-6-induced platelet adhesion to collagen beads remained unchanged throughout storage even though a small decrease could be detected on day 12 . The unit lacking swirling on day 12 showed a similar spontaneous platelet binding to beads, but in contrast to the other units, no increase in platelet adhesion to fibrinogen beads in response to TRAP-6 was observed. 


\section{A: Fibrinogen}
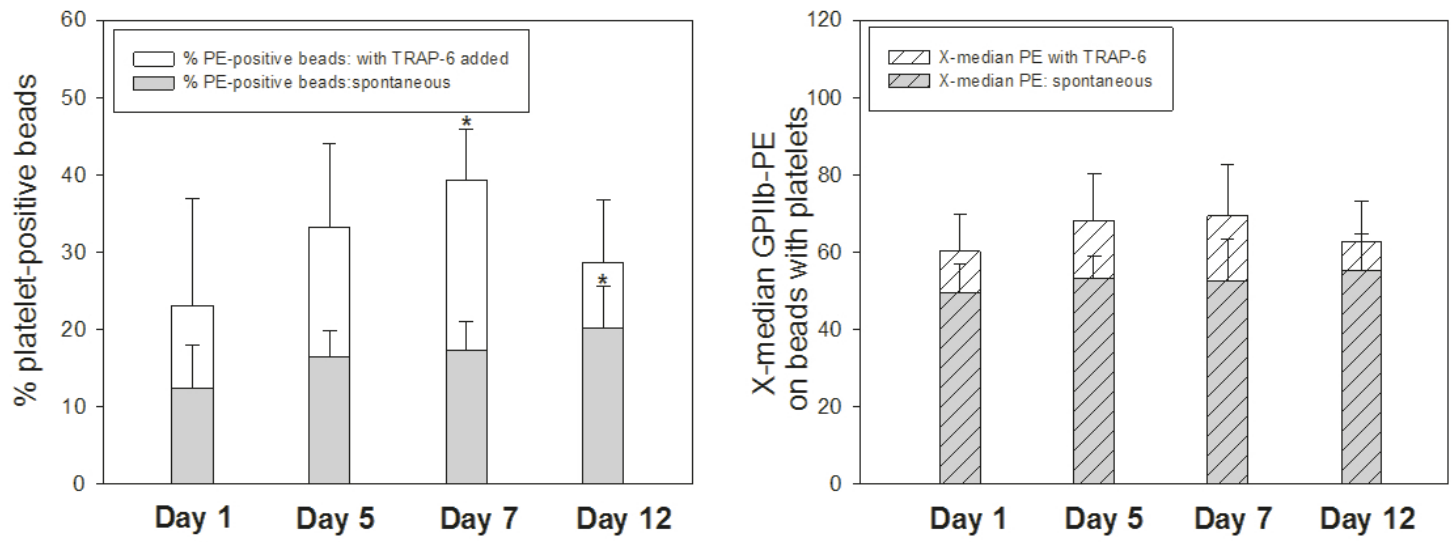

B: Collagen
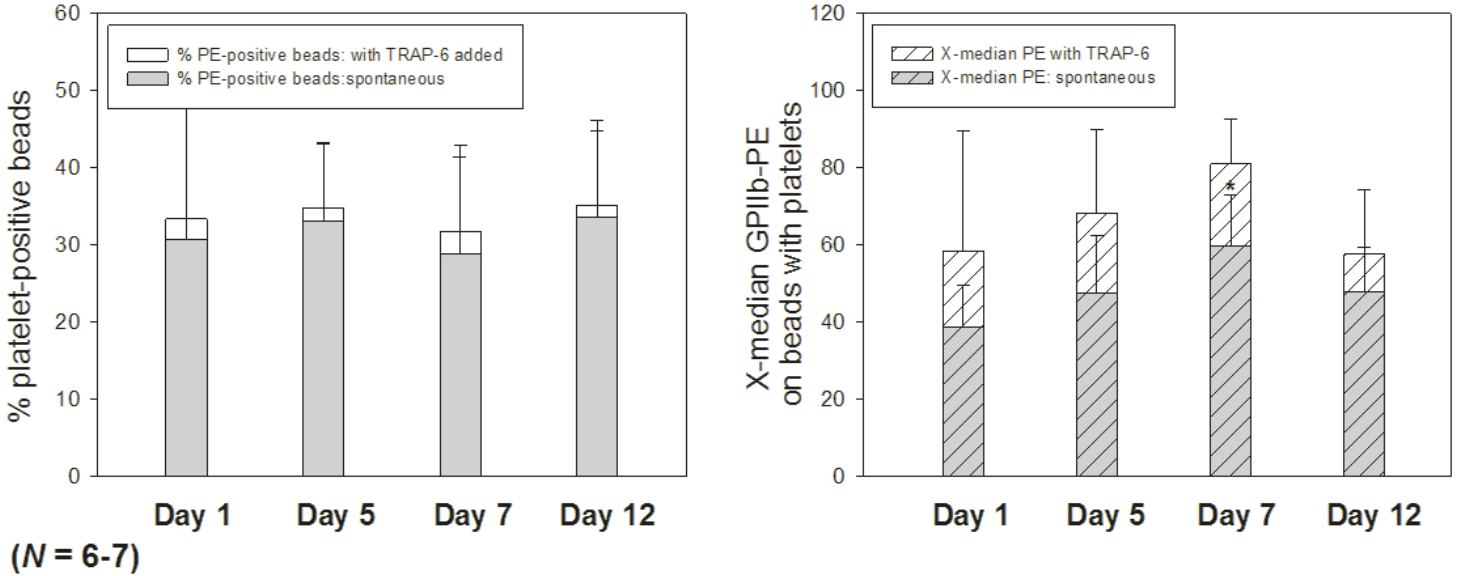

Figure 4. Adhesion of platelets to protein-coated beads during storage of PCs.

(A) Adhesion to fibrinogen-coated beads. (B) Adhesion to collagen-coated beads. Platelet adhesion is presented as percentage of GPIIb-positive (platelet-binding) beads (left) and as median GPIIb-PE fluorescence of the beads with platelets (right). Adhesion of non-activated platelets (grey bars) as well as TRAP-6-activated platelets (white bars) is presented. Results are presented as mean $+\mathrm{SD}$ for $N=7$ except on day 12 where $N=6$. * indicates $p<0.05$ as compared to day 1 .

\section{Expression of P-selectin and PS by platelets adhering to beads during storage of PCs}

The expression of P-selectin by platelets adhering to fibrinogen and collagen beads is

presented in Table III. The spontaneous expression did not change during storage. TRAP-6

caused a significant increase in the expression of P-selectin on platelets adhering to fibrinogen 
beads until day 7 ( $p<0.01$ ), but the increase was no longer significant on day 12 . TRAP-6 did not affect the expression of P-selectin on platelets adhering to collagen beads.

PS exposure on platelets adhering to fibrinogen beads had increased by day 12 (Table IV). In contrast, PS exposure on platelets adhering to collagen beads had decreased by day 7 but on day 12 the level was similar to day 1. TRAP-6 activation caused a significant increase in PS exposure on platelets adhering to fibrinogen beads until day $7(p<0.01)$, but no longer on day 12. Platelets from the unit with minimal swirling on day 12 showed a lower exposure of PS when adhering to collagen beads but the expression of P-selectin was similar to the other units.

Table III. Expression of P-selectin on platelets adhering to beads during PC storage

\begin{tabular}{|c|c|c|c|c|}
\hline & Day 1 & Day 5 & Day 7 & Day 12 \\
\hline $\begin{array}{l}\text { Fibrinogen beads } \\
\text { Non-activated PLTs }\end{array}$ & $6.7 \pm 0.7$ & $6.2 \pm 0.4$ & $6.2 \pm 0.6$ & $6.3 \pm 0.5(7.1)$ \\
\hline $\begin{array}{l}\text { Fibrinogen beads } \\
\text { TRAP- } 6 \text { activated PLTs }\end{array}$ & $7.9 \pm 0.8$ & $7.9 \pm 0.4$ & $7.7 \pm 0.7$ & $6.9 \pm 0.3 *(7.3)$ \\
\hline $\begin{array}{l}\text { Collagen beads } \\
\text { Non-activated PLTs }\end{array}$ & $18.4 \pm 1.7$ & $16.1 \pm 3.1$ & $13.4 \pm 2.7$ & $15.2 \pm 5.4(16.0)$ \\
\hline $\begin{array}{l}\text { Collagen beads } \\
\text { TRAP- } 6 \text { activated PLTs }\end{array}$ & $17.6 \pm 3.7$ & $15.9 \pm 3.3$ & $13.5 \pm 3.4$ & $15.2 \pm 4.1(16.6)$ \\
\hline
\end{tabular}

Expression of P-selectin determined on platelets adhering to fibrinogen- and collagen-coated beads in non-activated samples and TRAP- $6(30 \mu \mathrm{M})$-activated samples. P-selectin results are presented as median FITC fluorescence (arbitraty units). Results are shown as mean $\pm \mathrm{SD}$ for $N=7$ except on day 12 where $N=6$. The result for the PC with minimal swirling on day 12 is shown within parentheses. $*$ indicates $p<0.05$ as compared to day 1 . 
Table IV. Exposure of PS on platelets adhering to beads during PC storage

\section{Day $1 \quad$ Day $5 \quad$ Day $7 \quad$ Day 12}

Fibrinogen beads

$$
22 \pm 10 \quad 30 \pm 7 \quad 27 \pm 7 \quad 38 \pm 7 *(37)
$$

Non-activated PLTs

Fibrinogen beads $\quad 47 \pm 16 \quad 49 \pm 10 \quad 45 \pm 15 \quad 43 \pm 12(45)$

TRAP- 6 activated PLTs

Collagen beads

$$
78 \pm 21 \quad 77 \pm 12 \quad 57 \pm 12 * * \quad 74 \pm 16 \text { ( } 64)
$$

Non-activated PLTs

Collagen beads $\quad 82 \pm 14 \quad 75 \pm 14 \quad 63 \pm 12^{* *} \quad 78 \pm 12 \uparrow(42)$

TRAP- 6 activated PLTs

Exposure of PS as percentage of platelets adhering to fibrinogen- and collagen-coated beads in non-activated and TRAP-6 $(30 \mu \mathrm{M})$-activated samples being positive to annexin V. Results are shown as mean $\pm \mathrm{SD}$ for $N=7$ except on day 12 where $N=6$. The result for the PC with minimal swirling on day 12 is shown within parentheses. ${ }^{*}$ indicates $p<0.05,{ }^{* *}$ indicates $p<$ 0.01 as compared to day $1, \boldsymbol{\uparrow}$ indicates $p<0.05$ for day 7 as compared to day 12 . 


\section{Discussion}

Most researchers agree on the importance of measuring platelet adhesion, but there are several drawbacks with many of the currently available methods, including low throughput, cumbersome image analysis and low adhesion in the absence of red blood cells. Our aim was therefore to develop a flexible method allowing rapid evaluation of platelet adhesive capacity in blood or platelet suspensions. The developed method was used to examine adhesion of platelets from PCs during extended storage for 12 days.

Protein-coated beads have previously been used to study platelet expression of P-selectin or up-regulation of the fibrinogen receptor on adherent platelets [19]. However, it was not designed to quantify adhesion and activation of platelets simultaneously. Our method allows platelet adhesion to protein-coated beads to be evaluated with high throughput and rapid evaluation of a number of different variables. By diluting the samples, we minimise the interference of platelet aggregation in our adhesion assay. We used $20 \mu \mathrm{m}$ beads as they clearly separate from all other cellular blood components in the flow cytometer scatter plots and offer a larger surface for adhesion. In Figure 1, it may appear as the beads are disappearing in the upper right corner of the $\log / \log$ scatter plot, but the use of a lin/lin scatter plot to gate the single beads ensures that no beads are being excluded from analysis, and also allows exclusion of multiples of beads from analysis. For collagen beads, the presence of magnesium in the buffer is recommended, as we found a slightly lower adhesion in samples analysed for PS exposure, even though the changes upon activation and storage were comparable. This is also supported by the literature, where the presence of magnesium has been reported to be important for optimal platelet adhesion to collagen [20]. For this reason, Figure 4 only includes data from samples with magnesium in the buffer. 
In the whole blood experiments, beads coated with BSA showed low platelet adhesion without any signs of bead-induced platelet activation. Collagen beads showed moderate platelet adhesion unaffected by platelet activation or inhibition. Fibrinogen beads also showed moderate platelet adhesion, but in contrast to collagen, the adhesion increased significantly when platelets were activated by TRAP-6 or ADP and decreased when P2Y 12 or GPIIb/IIIa was inhibited, showing that adhesion to fibrinogen beads is sensitive to platelet activation or inhibition. This suggests that this assay could be useful in the evaluation of platelet inhibitors or to detect platelet activation ex vivo.

Platelet adhesion to BSA-coated beads remained low (approximately 2\%) throughout the storage period. Storage increased the platelets capacity to adhere to fibrinogen beads. However, it was not until after the normal shelf-life of PCs (5-7 days) that the increase in beads with adhered platelets became significant $(p<0.05$ on day 12). The collagen beads did not change in percentage of beads with adhering platelets, but did increase the amount of platelets adhering to each bead during the first 7 days of storage. There are few studies on platelet adhesion during storage and they vary in the method of platelet preparation, storage length and storage media, making direct comparisons difficult, as all these factors affect platelet function [6, 7, 9-11]. Furthermore these studies have tested platelets in presence of added red blood cells which could also influence their results.

In our study, stimulation with the platelet agonist TRAP-6 increased the adhesion of platelets to both fibrinogen and collagen beads, but for collagen beads, the changes were smaller. The platelet adhesion to fibrinogen beads in response to TRAP-6 increased with storage to day 7 but was followed by a decrease by day 12 . The reason for the changes in adhesion with storage is not clear but might be related to differences in expression of adhesion receptors on 
the platelet surface. The GPIIb/IIIa receptor is responsible for platelet binding to fibrinogen and aggregation. Several studies have shown a well preserved GPIIb/IIIa expression during storage $[6,21,22]$ but GPIIb/IIIa have also been reported to increase [23] and the receptor can exist in an activated or non-activated form, thus further complicating interpretations. Analysis of the active conformation of GPIIb/IIIa was not assessed in our study, which would have been of interest since there are studies reporting that the GPIIb/IIIa receptor undergoes changes with storage $[2,24]$, which might be one explanation to the increased platelet binding to fibrinogen beads observed in our study.

The expression of P-selectin on platelets adhering to fibrinogen or collagen beads was unaltered by storage. In general, platelets adhering to collagen-coated beads showed a higher P-selectin expression than the ones adhering to fibrinogen beads. PS exposure on platelets adhering to fibrinogen beads increased gradually with storage. A higher fraction of the collagen-binding platelets were exposing PS, which is consistent with other reports showing that adhesion to collagen leads to exposure of PS [8]. Exposure of PS on platelets adhering to collagen beads was significantly lower on day 7 as compared to day 1 , but then increased again at day 12, presumably connected to the spontaneous increase in PS exposure also detected on single platelets. TRAP-6 stimulation induced an increase in P-selectin and PS on platelets adhering to fibrinogen until day 7 but did not have any additional effect on the expression of P-selectin or PS on platelets adhering to collagen beads. The reduced response to TRAP-6 at the end of storage (lower fibrinogen binding and lower expression of P-selectin on adherent platelets) is in accordance to other studies using aggregometry to assess platelet function [25-27]. 
The single platelets showed no changes in GPIb expression as shown previously $[13,28]$ but there are also studies reporting a decrease in the expression of GPIb during storage [29, 30]. P-selectin increased with time as shown by us and others $[6,13,31]$. PS exposure increased only minimally during the normal shelf-life of PCs but by day 12 , the exposure had increased significantly. Others have also shown an increase in PS exposure during storage, but to various degrees $[6,16,18,30]$, the consequences of these apoptotic features during storage are largely unknown. Our results are in accordance with the results by Leytin et al. [32] that platelet activation is triggered before exposure of apoptotic features and our results support the hypothesis that apoptosis is triggered beyond the current shelf-life [1, 30]. It was also after day 7 that greatest changes in platelet adhesion and activation response occurred and they coincided with an increased rate in glucose consumption- and lactate formation.

One PC had only minimal swirling on day 12, presumably as glucose got depleted, although bacterial overgrowth cannot be ruled out as a contributing factor, as this was not investigated. This unit showed no increase in adhesion to fibrinogen after stimulation with TRAP-6 and the PS exposure of platelets adhering to collagen beads was lower despite a high spontaneous PS exposure, suggesting that platelets already showing signs of apoptosis are not capable of a proper activation response following adhesion to collagen beads.

There are some limitations with the described method. It shows a CV ranging between $8-20 \%$ for whole blood samples and $10-15 \%$ for PC samples, but this is comparable to what has been previously reported using perfusion chambers and cone and plate devices [12, 33], and as the sample preparation and analysis is rapid, duplicate or triplicate samples can be analysed. Another limitation is that our assay measures adhesion under less well defined, low shear conditions as compared to the use of flow chambers, but its simplicity makes it possible to use 
in clinical settings, where flow chambers would not be a feasible alternative. Furthermore this was a small in vitro study with large variation between PCs and the effects need to be elucidated in a larger clinical study.

Despite these limitations, our adhesion method offers a simple and user-friendly alternative to conventional adhesion methods and allows for rapid evaluation of a number of different variables. Furthermore we here show that our assay does not require presence of red blood cells and thus it allows for analysis of adhesion of platelets from concentrates without the requirement of sample manipulation by the addition of red blood cells as such have previously been shown to effect the results [12]. The method can be used for analysis of adhesion alone or of activation-induced adhesion in combination with analysis of activation/apoptosis markers following stimulation with different platelet agonists in both whole blood and PCs. The assay could be used to study platelet inhibitors and elucidate mechanisms involved in platelet adhesion of whole blood. The method could be used to study the adhesive properties of platelets in PCs following different preparation methods and storage variables. To be able to investigate potential subtle changes in platelet function during preparation and storage, it may be possible to increase the sensitivity and decrease the variance of the assay by modifications in protein coating, incubation conditions, shear, and other variables.

In conclusion, we describe a method that enables adhesion to be measured in platelet suspensions such as PCs. During storage of PCs, an increase in platelet adhesion and activation up to day 7 was observed, whereupon the activation response decreased. Our results suggest that the spontaneous adhesion is relatively well preserved during 12 days of storage, but that the capacity of activation-induced adhesion is decreasing. 


\section{Acknowledgements}

We thank our collaborators at the Department of Clinical Chemistry and Clinical Immunology and Transfusion Medicine at Linköping University Hospital for their assistance in the study. This study was supported by the County Council of Östergötland.

\section{Declaration of Interests}

This study was supported by the County Council of Östergötland.The authors report no declarations of interests. 


\section{References}

1. Cardigan R, Turner C, Harrison P. Current methods of assessing platelet function: relevance to transfusion medicine. Vox Sang 2005;88:153-163.

2. Cauwenberghs S, van Pampus E, Curvers J, Akkerman JW, Heemskerk JW. Hemostatic and signaling functions of transfused platelets. Transfus Med Rev 2007;21:287-294.

3. Ohto H, Nollet KE. Overview on platelet preservation: better controls over storage lesion. Transfus Apher Sci 2011;44:321-325.

4. Jilma-Stohlawetz P, Eichelberger B, Horvath M, Jilma B, Panzer S. In vitro platelet function of platelet concentrates prepared using three different apheresis devices determined by impedance and optical aggregometry. Transfusion 2009;49:1564-1568.

5. Bochsen L, Johansson PI, Kristensen AT, Daugaard G, Ostrowski SR. The influence of platelets, plasma and red blood cells on functional haemostatic assays. Blood Coagul Fibrinolysis 2011;22:167-175.

6. Curvers J, van Pampus EC, Feijge MA, Rombout-Sestrienkova E, Giesen PL, Heemskerk JW. Decreased responsiveness and development of activation markers of PLTs stored in plasma. Transfusion 2004;44:49-58.

7. Boomgaard MN, Gouwerok CW, Homburg CH, de Groot G, MJ IJ, de Korte D. The platelet adhesion capacity to subendothelial matrix and collagen in a flow model during storage of platelet concentrates for 7 days. Thromb Haemost 1994;72:611-616.

8. Cauwenberghs S, Feijge MA, Theunissen E, Heemskerk JW, van Pampus EC, Curvers J. Novel methodology for assessment of prophylactic platelet transfusion therapy by measuring increased thrombus formation and thrombin generation. Br J Haematol 2007;136:480-490.

9. Jilma-Stohlawetz P, Horvath M, Eichelberger B, Koren D, Jilma B, Panzer S. Platelet function under high-shear conditions from platelet concentrates. Transfusion 2008;48:129135. 
10. Horvath M, Eichelberger B, Koren D, Bohm A, Ay C, Jilma B, Panzer S, JilmaStohlawetz P. Function of platelets in apheresis platelet concentrates and in patient blood after transfusion as assessed by Impact-R. Transfusion 2010;50:1036-1042.

11. Morrison A, Hornsey VS, Prowse CV, Macgregor IR. Use of the DiaMed Impact R to test platelet function in stored platelet concentrates. Vox Sang 2007;93:166-172.

12. Morrison A, McMillan L, Hornsey VS, Prowse CV. Stored red-blood-cells inhibit platelet function under physiologic flow. Vox Sang 2010;99:362-368.

13. Tynngard N, Lindahl TL, Trinks M, Studer M, Berlin G. The quality of platelet concentrates produced by COBE Spectra and Trima Accel cell separators during storage for 7 days as assessed by in vitro methods. Transfusion 2008;48:715-722.

14. Bertolini F, Murphy S. A multicenter inspection of the swirling phenomenon in platelet concentrates prepared in routine practice. Biomedical Excellence for Safer Transfusion (BEST) Working Party of the International Society of Blood Transfusion. Transfusion 1996;36:128-132.

15. Andree HA, Reutelingsperger CP, Hauptmann R, Hemker HC, Hermens WT, Willems GM. Binding of vascular anticoagulant alpha (VAC alpha) to planar phospholipid bilayers. J Biol Chem 1990;265:4923-4928.

16. Albanyan AM, Harrison P, Murphy MF. Markers of platelet activation and apoptosis during storage of apheresis- and buffy coat-derived platelet concentrates for 7 days. Transfusion 2009;49:108-117.

17. Perrotta PL, Perrotta CL, Snyder EL. Apoptotic activity in stored human platelets. Transfusion 2003;43:526-535.

18. Saunders C, Rowe G, Wilkins K, Holme S, Collins P. In vitro storage characteristics of platelet concentrates suspended in 70\% SSP+(TM) additive solution versus plasma over a 14day storage period. Vox Sang 2011;101:112-121. 
19. Kao S, Turner NA, Moake JL, McIntire LV. A novel flow cytometric analysis for platelet activation on immobilized von Willebrand factor or fibrillar collagen. J Thromb Haemost 2003;1:347-354.

20. Whiss PA, Andersson RG. Divalent cations and the protein surface co-ordinate the intensity of human platelet adhesion and P-selectin surface expression. Blood Coagul Fibrinolysis 2002;13:407-416.

21. Metcalfe P, Williamson LM, Reutelingsperger CP, Swann I, Ouwehand WH, Goodall AH. Activation during preparation of therapeutic platelets affects deterioration during storage: a comparative flow cytometric study of different production methods. Br J Haematol 1997;98:86-95.

22. Sandgren P, Callaert M, Shanwell A, Gulliksson H. Storage of platelet concentrates from pooled buffy coats made of fresh and overnight-stored whole blood processed on the novel Atreus 2C+ system: in vitro study. Transfusion 2008;48:688-696.

23. Picker SM, Schneider V, Gathof BS. Platelet function assessed by shear-induced deposition of split triple-dose apheresis concentrates treated with pathogen reduction technologies. Transfusion 2009;49:1224-1232.

24. Thon JN, Devine DV. Translation of glycoprotein IIIa in stored blood platelets. Transfusion 2007;47:2260-2270.

25. Apelseth TO, Bruserud O, Wentzel-Larsen T, Bakken AM, Bjorsvik S, Hervig T. In vitro evaluation of metabolic changes and residual platelet responsiveness in photochemical treated and gamma-irradiated single-donor platelet concentrates during long-term storage. Transfusion 2007;47:653-665.

26. Picker SM, Oustianskaia L, Schneider V, Gathof BS. Functional characteristics of apheresis-derived platelets treated with ultraviolet light combined with either amotosalen-HCl (S-59) or riboflavin (vitamin B2) for pathogen-reduction. Vox Sang 2009;97:26-33. 
27. Thiele T, Iuga C, Janetzky S, Schwertz H, Gesell Salazar M, Furll B, Volker U, Greinacher A, Steil L. Early storage lesions in apheresis platelets are induced by the activation of the integrin alphaIIbbeta(3) and focal adhesion signaling pathways. J Proteomics 2012;76 Spec No.:297-315.

28. Albanyan AM, Murphy MF, Rasmussen JT, Heegaard CW, Harrison P. Measurement of phosphatidylserine exposure during storage of platelet concentrates using the novel probe lactadherin: a comparison study with annexin V. Transfusion 2009;49:99-107.

29. Sandgren P, Hansson M, Gulliksson H, Shanwell A. Storage of buffy-coat-derived platelets in additive solutions at 4 degrees $\mathrm{C}$ and 22 degrees $\mathrm{C}$ : flow cytometry analysis of platelet glycoprotein expression. Vox Sang 2007;93:27-36.

30. Cookson P, Sutherland J, Turner C, Bashir S, Wiltshire M, Hancock V, Smith K, Cardigan R. Platelet apoptosis and activation in platelet concentrates stored for up to 12 days in plasma or additive solution. Transfus Med 2010;20:392-402.

31. Zhang JG, Carter CJ, Culibrk B, Devine DV, Levin E, Scammell K, Weiss S, Gyongyossy-Issa MI. Buffy-coat platelet variables and metabolism during storage in additive solutions or plasma. Transfusion 2008;48:847-856.

32. Leytin V, Allen DJ, Mutlu A, Mykhaylov S, Lyubimov E, Freedman J. Platelet activation and apoptosis are different phenomena: evidence from the sequential dynamics and the magnitude of responses during platelet storage. Br J Haematol 2008;142:494-497.

33. Hansen RR, Wufsus AR, Barton ST, Onasoga AA, Johnson-Paben RM, Neeves KB. High content evaluation of shear dependent platelet function in a microfluidic flow assay. Annals of biomedical engineering 2013;41:250-262. 